\title{
openheart Plasma brain natriuretic peptide concentrations in patients with valvular heart disease
}

\author{
Vishal Sharma, ${ }^{1,2}$ Ralph A Stewart, ${ }^{3}$ Mildred Lee, ${ }^{4}$ Ruvin Gabriel, ${ }^{4}$ Niels Van Pelt, ${ }^{4}$ \\ David E Newby, ${ }^{2}$ Andrew J Kerr ${ }^{4}$
}

To cite: Sharma V, Stewart RA, Lee M, et al. Plasma brain natriuretic peptide concentrations in patients with valvular heart disease. Open Heart 2016;3: e000184. doi:10.1136/ openhrt-2014-000184

Received 12 August 2014 Revised 28 March 2015 Accepted 3 June 2015

\section{(1) Corossark}

${ }^{1}$ Department of Cardiology, Royal Liverpool University Hospital, Liverpool, UK ${ }^{2}$ British Heart Foundation Centre for Cardiovascular Science, University of Edinburgh, Edinburgh, UK

${ }^{3}$ Green Lane Cardiovascular Service, Auckland City Hospital, Auckland, New Zealand

${ }^{4}$ Department of Cardiology, Middlemore Hospital, Auckland, New Zealand

Correspondence to Dr Vishal Sharma; vishalsharma76@gmail.com

\section{ABSTRACT}

Objective: Plasma brain natriuretic peptide (BNP) concentrations predict prognosis in patients with valvular heart disease (VHD), but it is unclear whether this directly relates to disease severity. We assessed the relationship between BNP and echocardiographic measures of disease severity in patients with VHD.

Methods: Plasma BNP concentrations were measured in patients with normal left ventricular (LV) systolic function and isolated VHD (mitral regurgitation (MR), $\mathrm{n}=33$; aortic regurgitation (AR), $\mathrm{n}=39$; aortic stenosis (AS), $n=34$; mitral stenosis (MS), $n=30$ ), and agematched and sex-matched controls $(n=39)$ immediately prior to exercise stress echocardiography.

Results: Compared with controls, patients with VHD had elevated plasma BNP concentrations (MR median 35 (IQR 23-52), AR 34 (22-45), AS 31 (22-60), MS 58 (34-90); controls $24(16-33) \mathrm{pg} / \mathrm{mL} ; \mathrm{p}<0.01$ for all). LV end diastolic volume index varied by valve lesion; (MR (mean $77 \pm 14)$, AR $(91 \pm 28)$, AS $(50 \pm 17)$, MS (43 \pm 11$)$, controls $\left.(52 \pm 13) \mathrm{mL} / \mathrm{m}^{2} ; \mathrm{p}<0.0001\right)$. There were no associations between LV volume and BNP. Left atrial $(L A)$ area index varied (MR $\left(18 \pm 4 \mathrm{~cm}^{2} / \mathrm{m}^{2}\right)$, AR $(12 \pm 2)$, AS $(11 \pm 3)$, MS $(19 \pm 6)$, controls (11 \pm 2$) ; p<0.0001)$, but correlated with plasma BNP concentrations: MR $(r=0.42, p=0.02), M S(r=0.86$, $p<0.0001), A R(r=0.53, p=0.001), A S(r=0.52$, $\mathrm{p}=0.002$ ). Higher plasma BNP concentrations were associated with increased pulmonary artery pressure and reduced exercise capacity. Despite adverse cardiac remodelling, $81(60 \%)$ patients had a BNP concentration within the normal range.

Conclusions: Despite LV remodelling, plasma BNP concentrations are often normal in patients with VHD. Conversely, mild elevations of BNP occur with LA dilatation in the presence of normal LV. Plasma BNP concentrations should be interpreted with caution when assessing patients with VHD.

\section{INTRODUCTION}

Brain natriuretic peptide (BNP) is a powerful prognostic marker in patients with a broad range of cardiac diseases, including acute ${ }^{1}$ and chronic heart failure, ${ }^{23}$ and postmyocardial infarction. ${ }^{4}$ Plasma levels of BNP may

\section{KEY QUESTIONS}

What is already known about this subject?

- It is recognised that plasma brain natriuretic peptide (BNP) concentrations are elevated in patients with different valvular heart lesions. Patients who are symptomatic or have more severe valve lesions tend to have higher plasma BNP concentrations. Consequently, for a given valve lesion, it has been suggested that BNP levels could be used to screen for significant valve disease, or identify patients with an adverse prognosis. Many of these studies were small and did not include any objective assessment of functional capacity. In addition, no prior study has systematically compared plasma BNP concentrations across different valve lesions.

What does this study add?

- This study recruited a relatively large number of patients with valvular heart disease, in order to assess plasma BNP levels across different valve lesions. The main findings are that significant adverse cardiac remodelling can occur in valvular heart disease despite plasma BNP concentrations that remain within the normal range. In addition, an elevated BNP was found in a number of asymptomatic patients, and these patients performed less well on exercise testing than those whose plasma BNP concentration remained within the normal range. Finally, plasma BNP concentrations in all valve lesions were associated with markers of left atrial but not left ventricular size suggesting that left atrial release of BNP is significant in patients with valvular heart disease.

How might this impact on clinical practice?

- As significant cardiac remodelling can occur despite plasma BNP concentrations that remain within the normal range, plasma BNP levels should be interpreted with caution and not used as a screening test to detect significant valve disease. However, despite this, in an apparently asymptomatic patient, an elevated BNP is predictive of poor performance on exercise testing, and this finding should prompt careful reassessment of the patient. 
also increase in patients with valvular heart disease, ${ }^{5-12}$ particularly in patients with symptoms and more severe valve lesions. Higher levels of BNP may be associated with a worse outcome in patients with mitral regurgitation $^{13}$ and aortic stenosis. ${ }^{14}$ Patients with valve disease who have significant left ventricular (LV) impairment often have very high levels of $\mathrm{BNP}^{15}{ }^{16}$ but in these patients the indications for surgery are already clear. Measurement of BNP may, however, be useful in patients with heart valve disease and preserved LV systolic function, especially when symptoms and haemodynamic data are equivocal.

Previous studies have evaluated the relationship between plasma BNP concentrations and echocardiographic measures of disease severity, as well as clinical outcomes for specific valve lesions including aortic stenosis (AS), aortic regurgitation (AR), mitral regurgitation and mitral stenosis (MS). ${ }^{5} \quad 9 \quad 17 \quad 18$ However, each aortic and mitral valve lesion causes different cardiac pressure and volume loads, and it is unclear whether the mechanism of BNP release and clinical implications of elevated plasma BNP concentrations are dependent on valve lesion type. These previous studies did not directly compare patients with different valve lesions, which result in different volume and pressure loads on the heart. The aim of this study was to identify both common and valve lesion-specific factors associated with higher plasma BNP concentrations in patients with preserved LV systolic function, who have isolated AS, AR, mitral regurgitation (MR) or MS, and to assess suitability of BNP for identifying patients with severe valvular heart disease (VHD) across different valve lesions.

\section{METHODS}

Patients with moderate to severe AS (peak velocity $>3.0 \mathrm{~m} / \mathrm{s}$ ), moderate to severe AR (left ventricular outflow tract (LVOT) width ratio $>0.3$ and holodiastolic flow reversal in descending thoracic aorta), moderate to severe MS (mitral valve area $<1.5 \mathrm{~cm}^{2}$ ), and moderate to severe or severe MR (effective regurgitant orifice area $>0.3 \mathrm{~cm}^{2}$ ) were identified from echocardiogram reports and outpatient clinics in the Auckland region. ${ }^{19}{ }^{20}$ All patients had normal left ventricular ejection fraction (LVEF) $\quad(\mathrm{AS} \geq 50 \%, \quad$ AR $>50 \%, \quad \mathrm{MR} \geq 60 \%, \quad \mathrm{MS} \geq 50) .{ }^{21}$ Exclusion criteria included ischaemic heart disease, significant renal impairment (creatinine $>0.16 \mathrm{mmol} / \mathrm{L}$ on baseline blood tests performed at enrolment), respiratory disease, and an inability to walk on a treadmill or a contraindication to exercise testing. All patients eligible for inclusion were invited to participate. The patient's symptomatic status was assessed according to the New York Heart Association (NYHA) criteria by a cardiologist blinded to echocardiographic data and peptide levels at the time of enrolment. Patients who were mildly symptomatic, or who had equivocal symptoms, were considered to be NYHA 2 in the analysis. Control subjects were volunteers from the local community who were age and sex matched. They were recruited by advertisement and had no clinical evidence of cardiovascular or respiratory disease. The study was approved by the Northern X Ethics Committee, Auckland. The authors of this manuscript have certified that they comply with the Principles of Ethical Publishing. ${ }^{22}$ The main outcome was the ability of plasma BNP concentrations to identify patients with haemodynamically significant valve disease as assessed by exercise stress echocardiography.

\section{Exercise testing}

All patients underwent symptom-limited exercise testing on a motorised treadmill. A standard Bruce protocol was used for patients with mitral regurgitation. However, it was noted that there was significant clustering of exercise times at the end of each stage, and this was felt to adversely affect the objectivity of the determination of exercise capacity. Consequently for subsequent patients, the protocol was adjusted so that the speed and/or incline of the treadmill were increased at $1 \mathrm{~min}$ intervals. The maximum workload at the end of each 3 min stage remained equivalent to that of the standard Bruce protocol. Exercise was stopped for significant dyspnoea, chest discomfort, presyncope, fatigue or at patient request.

\section{Echocardiography}

All echocardiograms were performed by experienced sonographers on cardiac ultrasound scanners (Vivid 7, General Electric, Vingmed Ultrasound, Norway). All patients underwent comprehensive examination including M-mode, two-dimensional, Doppler and tissue Doppler echocardiography modified according to their underlying valvular lesions consistent with current guidelines. ${ }^{20}{ }^{23}$ All analyses were performed offline (Echopac PC, GE Medical, Milwaukee, Wisconsin, USA) by an experienced cardiologist blinded to the results of the patient characteristics and BNP data. All measurements were averaged from at least three, or in cases of atrial fibrillation (AF), five cardiac cycles. The LV end-systolic and end-diastolic volumes and EF were measured from the apical four-chamber view using the modified Simpson's single-plane method. ${ }^{24}$ This method was chosen to allow comparison with the postexercise volumes. The left atrial area was measured in the apical four-chamber view. ${ }^{25}$

Quantitative and qualitative measures of AS and regurgitation severity were made according to American Society of Echocardiography guidelines. $^{19}{ }^{20}$ Quantitative measures of AS severity included peak velocity, mean pressure drop and aortic valve area. Assessment of AR severity included the AR jet:LVOT width ratio, AR pressure half time, peak diastolic and end-diastolic flow velocities in the descending thoracic and abdominal aorta.

The severity of MR was assessed by quantitative Doppler with mitral and aortic stroke volumes, ${ }^{26}$ and by the proximal isovelocity surface area method. ${ }^{27}$ The stroke volumes obtained from the two methods were 
averaged to give a mean regurgitant volume. ${ }^{11}$ The mitral valve area (MVA) was obtained using direct planimetry, pressure half time and continuity methods. ${ }^{28-30}$ The median MVA from these three methods was used for analysis. Mean transmitral pressure gradients were obtained by tracing the continuous wave Doppler signal across the mitral valve. ${ }^{31}$

To facilitate estimation of right ventricular systolic pressure, agitated saline was injected to enhance the tricuspid regurgitation profile. ${ }^{32}$ The peak pulmonary artery systolic pressure was derived using the simplified Bernouilli equation from the peak tricuspid regurgitant jet, and added to an estimate of right atrial pressure obtained from imaging of the inferior vena cava. ${ }^{33}$ Left atrial area, left ventricular volumes, valve areas and effective regurgitant orifice areas were indexed to body surface area. ${ }^{34}$

\section{Measurement of natriuretic peptides}

Blood samples were obtained from an indwelling intravenous catheter after 15 min lying supine, immediately prior to the baseline echocardiogram. Patients fasted for $2 \mathrm{~h}$ prior to the investigations. Blood samples were collected in EDTA tubes, and plasma stored at $-80^{\circ} \mathrm{C}$ and for measurement of BNP levels at the study end using established radioimmunoassays. ${ }^{35}$ The upper limit of the normal reference range for $\mathrm{BNP}$ is $42 \mathrm{pg} / \mathrm{mL}$. To convert BNP levels expressed in $\mathrm{pg} / \mathrm{mL}$ to $\mathrm{pmol} / \mathrm{L}$, divide by $3.47 .^{35} 36$

\section{Statistical analysis}

Previous studies have demonstrated a mean plasma BNP concentration of $22 \pm 7 \mathrm{pg} / \mathrm{mL}$ in the normal healthy population. ${ }^{35} \mathrm{~A}$ minimum sample size of 30 in each valve subgroup was required to detect a $20 \%$ increase in plasma BNP concentrations compared to controls (power of $80 \%$ and $\alpha=0.01$ ). Categorical data were presented as frequency and percentage, and continuous data were presented as mean $\pm \mathrm{SD}$, or median and $\mathrm{IQR}$. BNP underwent natural $\log$ transformation in analyses due to its right skewed distributions unless specified, and was reported as median and interquartile range. Comparison of all valves versus the control group and valve subgroups, was performed using the $\chi^{2}$ or Fisher's exact tests for categorical data where appropriate. For continuous variables, two independent sample Student t test or non-parametric Mann-Whitney $\mathrm{U}$ test was used where appropriate, for comparing all-valve versus control groups. For comparison between subgroups of valves, one-way analysis of variance was performed followed by the Dunnett's test, to compare each valve subgroup with control subjects. The Kruskal-Wallis test was used when the continuous data was not normally distributed. Pearson correlation coefficients were reported for linear associations. All $\mathrm{p}$ values reported were two-tailed, and $\mathrm{a} p$ value $<0.05$ was considered significant. Data were analysed using SAS statistical package, V.9.1.3 (SAS Institute, Cary, North Carolina, USA).

\section{RESULTS}

All patients who agreed to participate completed all aspects of the study. One hundred and seventy-five subjects with isolated MR $(n=33)$, AR $(n=39)$, AS $(n=34)$ and MS $(n=30)$ or normal valves $(n=39)$ were studied. Plasma BNP concentrations and exercise treadmill data were available for all patients. Plasma BNP concentrations (median, interquartile range) were elevated in all valve lesions compared with controls (MR $(35 \mathrm{pg} / \mathrm{mL}$, 23-52), AR (34 pg/mL, 22-45), AS (31 pg/mL, 22-60), MS (58 pg/mL, 34-90), control (24 pg/mL, 16-33); $\mathrm{p}<0.01$ for all $)$.

Patients with AS were predominantly male, whereas patients with MS were predominantly female (table 1). In keeping with the higher proportion of degenerative valve disease, patients with AS were generally older than those with other valve lesions. Patients with mitral valve disease had a higher plasma BNP concentration than those with aortic valve disease $(46(29-73)$ vs $32(22-49) \mathrm{pg} / \mathrm{mL}$, $\mathrm{p}=0.012$ ). For each given valve lesion, patients with MS had the highest plasma concentration of BNP. This remained the case if patients with $\mathrm{AF}$ were excluded from the analysis (BNP in MS with sinus rhythm 55 (30-73) vs other valve lesions $34(22-52) \mathrm{pg} / \mathrm{mL} ; \mathrm{p}=0.02)$.

The relative use of cardiac medication differed between valve groups. Patients with AR were the most likely to be taking medication, with $64 \%$ of patients taking an ACE inhibitors inhibitor. Forty-three per cent of patients with MS were taking a loop diuretic (at a dose of $40 \mathrm{mg}$ furosemide or less), and $32 \%$ of patients with AS were taking a betablocker. Patients with AS who were not taking a betablocker had lower plasma BNP concentrations than those who were $(26(20,41)$ vs 42 $(34,63) ; \mathrm{p}=0.03)$. There was no other statistically significant difference in plasma BNP concentrations between patients who were taking medication compared to those who were not in any other valve subgroup.

\section{Adverse cardiac remodelling}

Although all subjects had normal LVEF, LV end diastolic volume index (LVEDVI) varied considerably across patients and by valve lesion (MR $77 \pm 14$, AR $91 \pm 28$, AS $50 \pm 17$, MS $43 \pm 11$ and controls $52 \pm 13 \mathrm{~mL} / \mathrm{m}^{2} ; \mathrm{p}<0.0001$ ). For each valvular lesion, patients were divided into two groups depending on whether the plasma BNP concentration was within the normal range $(\leq 42 \mathrm{pg} / \mathrm{mL})$ or elevated. The relative numbers of patients with elevated BNP in each group are shown in table 2. Even when plasma BNP concentration remained within the normal range, those with regurgitant valve lesions had significant LV dilatation compared to controls: (LVEDVI in MR $78 \pm 14$, AR $88 \pm 27$ and controls $52 \pm 12 \mathrm{~mL} / \mathrm{m}^{2}$; $\mathrm{p}=<0.0001)$. There was no association between any resting measure of LV function and plasma BNP concentration (table 3).

LA area index (LAAI) was greater in patients with

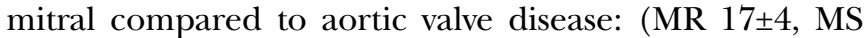
$19 \pm 6$, AR $11 \pm 2$, AS $11 \pm 2)$ and controls $\left(11 \pm 2 \mathrm{~cm}^{2} / \mathrm{m}^{2}\right.$; 
Table 1 Baseline characteristics, baseline echocardiographic and exercise data

\begin{tabular}{|c|c|c|c|c|c|c|c|c|}
\hline & Controls & MR & MS & AS & AR & All valves & p Value* & p Valuet \\
\hline Number of subjects & 39 & 33 & 30 & 34 & 39 & 136 & & \\
\hline Age (years) & $55 \pm 15$ & $53 \pm 16$ & $49 \pm 14$ & $67 \pm 9 \ddagger$ & $42 \pm 14 \ddagger$ & $53 \pm 16$ & 0.404 & $<0.001$ \\
\hline Female, n (\%) & $16(41)$ & $15(45)$ & $28(93)$ & $4(12)$ & $6(15)$ & $53(39)$ & 0.817 & $<0.001$ \\
\hline Body mass index $\left(\mathrm{kg} / \mathrm{m}^{2}\right)$ & $25.8 \pm 3.5$ & $27.7 \pm 5.0$ & $28.6 \pm 5.8$ & $28.9 \pm 4.5 \ddagger$ & $28.8 \pm 4.7 \ddagger$ & $28.5 \pm 4.9$ & 0.001 & 0.023 \\
\hline Atrial fibrillation, $\mathrm{n}(\%)$ & $0(0)$ & $0(0)$ & $8(27)$ & $0(0)$ & $0(0)$ & $8(6)$ & 0.202 & $<0.001$ \\
\hline Symptomatic, n (\%) & $1(3)$ & $17(52)$ & $14(47)$ & $17(50)$ & $7(18)$ & $55(40)$ & $<0.001$ & $<0.001$ \\
\hline \multicolumn{9}{|l|}{ Medication, $\mathrm{n}(\%)$} \\
\hline Loop diuretic & $0(0)$ & $4(12)$ & $13(43)$ & $5(15)$ & $2(5)$ & $24(18)$ & 0.003 & $<0.001$ \\
\hline ACE inhibitor & $0(0)$ & $6(18)$ & $4(13)$ & $4(12)$ & $25(64)$ & $39(29)$ & $<0.001$ & $<0.001$ \\
\hline Beta blocker & $0(0)$ & $6(18)$ & $4(13)$ & $11(32)$ & $2(5)$ & $23(17)$ & 0.006 & $<0.001$ \\
\hline BNP $(\mathrm{pg} / \mathrm{mL})$ & $24(16-33)$ & $35(23-52)$ & $58(34-90)$ & $31(22-60)$ & $34(22-45)$ & $36(24-62)$ & $<0.001$ & $<0.001$ \\
\hline Left atrial area index & $10.5 \pm 1.9$ & $17.5 \pm 4.4 \ddagger$ & $19.3 \pm 5.9 \ddagger$ & $11.5 \pm 2.5$ & $10.8 \pm 2.0$ & $14.5 \pm 5.4$ & $<0.001$ & $<0.001$ \\
\hline LVEDVI $\left(\mathrm{mL} / \mathrm{m}^{2}\right)$ & $52.3 \pm 12.7$ & $76.6 \pm 14.0 \ddagger$ & $43.4 \pm 11.2$ & $49.6 \pm 16.5$ & $91.3 \pm 28.4 \ddagger$ & $66.9 \pm 27.6$ & 0.003 & $<0.001$ \\
\hline LVESVI $\left(\mathrm{mL} / \mathrm{m}^{2}\right)$ & $19.0 \pm 7.1$ & $26.9 \pm 6.5 \ddagger$ & $16.5 \pm 5.9$ & $20.8 \pm 12.3$ & $33.9 \pm 14.1 \ddagger$ & $25.1 \pm 12.4$ & 0.005 & $<0.001$ \\
\hline LVEF (\%) & $64.5 \pm 7.6$ & $64.6 \pm 6.7$ & $62.4 \pm 7.3$ & $61.9 \pm 7.0$ & $63.7 \pm 6.3$ & $63.2 \pm 6.8$ & 0.320 & 0.380 \\
\hline PA pressure $(\mathrm{mm} \mathrm{Hg})$ & $24 \pm 4$ & $37 \pm 13 \ddagger$ & $36 \pm 12 \ddagger$ & $32 \pm 7 \ddagger$ & $28 \pm 7$ & $33 \pm 10$ & $<0.001$ & $<0.001$ \\
\hline Peak heart rate (bpm) & $153 \pm 16$ & $153 \pm 21$ & $148 \pm 24$ & $129 \pm 20 \ddagger$ & $133 \pm 19 \ddagger$ & $140 \pm 23$ & 0.0003 & $<0.001$ \\
\hline Exercise capacity (METS) & $12.5 \pm 3.1$ & $9.1 \pm 2.7 \ddagger$ & $6.3 \pm 2.6 \ddagger$ & $9.1 \pm 3.2 \ddagger$ & $10.9 \pm 3.4$ & $9.0 \pm 3.4$ & $<0.001$ & $<0.001$ \\
\hline Peak systolic BP (mm Hg) & $169 \pm 23$ & $163 \pm 30$ & $132 \pm 23 \ddagger$ & $159 \pm 22$ & $188 \pm 24 \ddagger$ & $163 \pm 32$ & 0.183 & $<0.001$ \\
\hline
\end{tabular}

${ }^{*} \mathrm{p}$ Value relates to all-valves patients compared with controls.

${ }^{*} \mathrm{p}$ Value relates to all-valves patients compare
tp Value for comparison between subgroups.

$\ddagger$ Comparison significant at the 0.05 level between each valve group versus controls.

Results are Mean \pm SD except BNP which is median (IQR).

BNP, brain natriuretic peptide; BP, blood pressure; LVEDVI, left ventricular end-diastolic volume index; LVEF, left ventricular ejection fraction; LVESVI, left ventricular end-systolic volume index;

METS, metabolic equivalents; PA, pulmonary artery. 
Table 2 Measures of cardiac remodelling, valve severity and exercise capacity for each valve lesion stratified by BNP

\begin{tabular}{|c|c|c|c|c|c|c|}
\hline & & Controls & $\begin{array}{l}\text { Aortic } \\
\text { stenosis }\end{array}$ & $\begin{array}{l}\text { Mitral } \\
\text { stenosis }\end{array}$ & $\begin{array}{l}\text { Aortic } \\
\text { regurgitation }\end{array}$ & $\begin{array}{l}\text { Mitral } \\
\text { regurgitation }\end{array}$ \\
\hline \multirow[t]{3}{*}{ Number of subjects } & Normal BNP & 34 & 24 & 10 & 29 & 21 \\
\hline & High BNP & 5 & 10 & 20 & 10 & 12 \\
\hline & Total & 39 & 34 & 30 & 29 & 33 \\
\hline \multirow[t]{3}{*}{ NYHA class $2, \mathrm{n}(\%)$} & Normal BNP & $1(2.9)$ & 8 (33.3) & 3 (33.3) & $3(10.3)$ & 7 (33.3) \\
\hline & High BNP & $0(0)$ & $7(70.0)$ & $11(55.0)$ & $2(20.0)$ & $8(66.7)$ \\
\hline & $\mathrm{p}$ Value & 1.00 & 0.20 & 0.26 & 0.03 & 0.09 \\
\hline \multirow[t]{3}{*}{ Severe valve disease, $\mathrm{n}(\%)$} & Normal BNP & - & $3(13)$ & $1(10)$ & $15(52)$ & $15(71)$ \\
\hline & High BNP & - & $5(50)$ & $4(20)$ & $9(90)$ & $6(50)$ \\
\hline & $p$ Value & - & 0.03 & 0.64 & 0.06 & 0.27 \\
\hline \multirow[t]{3}{*}{ LV end-diastolic volume index $\left(\mathrm{mL} / \mathrm{m}^{2}\right)$} & Normal BNP & $52 \pm 12$ & $50 \pm 17$ & $45 \pm 7$ & $88 \pm 27$ & $78 \pm 14$ \\
\hline & High BNP & $53 \pm 16$ & $48 \pm 17$ & $42 \pm 13$ & $100 \pm 31$ & $75 \pm 14$ \\
\hline & $\mathrm{p}$ Value & 0.96 & 0.73 & 0.50 & 0.20 & 0.59 \\
\hline \multirow[t]{2}{*}{ LV end-systolic volume index $\left(\mathrm{mL} / \mathrm{m}^{2}\right)$} & Normal BNP & $19 \pm 7$ & $19 \pm 9$ & $16 \pm 5$ & $32 \pm 13$ & $28 \pm 6$ \\
\hline & $\begin{array}{l}\text { High BNP } \\
\text { p Value }\end{array}$ & $19 \pm 60.89$ & $24 \pm 190.95$ & $16 \pm 60.91$ & $39 \pm 170.25$ & $26 \pm 70.81$ \\
\hline \multirow[t]{3}{*}{ LV ejection fraction (\%) } & Normal BNP & $64 \pm 8$ & $61 \pm 7$ & $63 \pm 8$ & $64 \pm 6$ & $64 \pm 7$ \\
\hline & High BNP & $65 \pm 9$ & $63 \pm 6$ & $62 \pm 7$ & $63 \pm 6$ & $66 \pm 7$ \\
\hline & $\mathrm{p}$ Value & 0.90 & 0.47 & 0.57 & 0.54 & 0.57 \\
\hline \multirow[t]{3}{*}{$\mathrm{LA}$ area index $\left(\mathrm{cm}^{2} / \mathrm{m}^{2}\right)$} & Normal BNP & $10 \pm 2$ & $11 \pm 2$ & $15 \pm 2$ & $10 \pm 2$ & $17 \pm 4$ \\
\hline & High BNP & $11 \pm 3$ & $13 \pm 3$ & $21 \pm 6$ & $12 \pm 2$ & $18 \pm 5$ \\
\hline & $\mathrm{p}$ Value & 0.69 & 0.008 & 0.0005 & 0.07 & 0.47 \\
\hline \multirow[t]{3}{*}{ Pulmonary artery pressure $(\mathrm{mm} \mathrm{Hg})$} & Normal BNP & $24 \pm 4$ & $31 \pm 6$ & $34 \pm 16$ & $27 \pm 6$ & $32 \pm 7$ \\
\hline & High BNP & $25 \pm 6$ & $35 \pm 7$ & $36 \pm 9$ & $32 \pm 6$ & $47 \pm 16$ \\
\hline & $\mathrm{p}$ Value & 0.77 & 0.16 & 0.14 & 0.06 & 0.008 \\
\hline \multirow[t]{3}{*}{ Valve area index/EROA index $\left(\mathrm{cm}^{2} / \mathrm{m}^{2}\right)$} & Normal BNP & - & $0.51 \pm 0.13$ & $0.75 \pm 0.15$ & $0.10 \pm 0.07$ & $0.34 \pm 0.16$ \\
\hline & High BNP & - & $0.41 \pm 0.09$ & $0.62 \pm 0.14$ & $0.16 \pm 0.08$ & $0.32 \pm 0.16$ \\
\hline & $\mathrm{p}$ Value & & 0.04 & 0.03 & 0.02 & 0.72 \\
\hline \multirow[t]{3}{*}{ Exercise capacity (METS), } & Normal BNP & $12 \pm 3$ & $10 \pm 3$ & $8 \pm 3$ & $11 \pm 4$ & $10 \pm 2$ \\
\hline & High BNP & $14 \pm 3$ & $7 \pm 2$ & $5 \pm 2$ & $11 \pm 3$ & $7 \pm 2$ \\
\hline & pValue & 0.22 & 0.003 & 0.02 & 0.65 & 0.001 \\
\hline
\end{tabular}

All data is mean $\pm S D$ unless otherwise stated. $p$ Values relate to comparison of normal versus raised BNP groups.

BNP, brain natriuretic peptide; EROA, effective regurgitant orifice area; LA, left atrium; LV, left ventricle; METS, metabolic equivalents; NYHA, New York Heart Association.

Table 3 Associations between BNP, echocardiographic variables and exercise capacity

\begin{tabular}{|c|c|c|c|c|c|c|}
\hline & $\begin{array}{l}\text { Controls r } \\
\text { (p) }\end{array}$ & $\begin{array}{l}\text { Aortic } \\
\text { stenosis r } \\
\text { (p) }\end{array}$ & $\begin{array}{l}\text { Mitral } \\
\text { stenosis } r(p)\end{array}$ & $\begin{array}{l}\text { Aortic } \\
\text { regurgitation r } \\
\text { (p) }\end{array}$ & $\begin{array}{l}\text { Mitral } \\
\text { regurgitation r } \\
\text { (p) }\end{array}$ & All valves $r(p)$ \\
\hline $\begin{array}{l}\text { LV end-diastolic volume } \\
\text { index }\left(\mathrm{mL} / \mathrm{m}^{2}\right)\end{array}$ & $0.22(0.20)$ & $0.02(0.93)$ & $-0.24(0.20)$ & $0.16(0.34)$ & $-0.02(0.28)$ & $-0.01(0.85)$ \\
\hline $\begin{array}{l}\text { LV end-systolic volume } \\
\text { index }\left(\mathrm{mL} / \mathrm{m}^{2}\right)\end{array}$ & $0.13(0.43)$ & $0.10(0.59)$ & $-0.06(0.74)$ & $0.19(0.24)$ & $-0.19(0.31)$ & $0.01(0.86)$ \\
\hline $\begin{array}{l}\text { Left atrial area index } \\
\left(\mathrm{cm}^{2} / \mathrm{m}^{2}\right)\end{array}$ & $0.10(0.57)$ & $0.52(0.002)$ & $0.86(<0.0001)$ & $0.53(0.001)$ & $0.42(0.02)$ & $0.57(<0.0001)$ \\
\hline $\begin{array}{l}\text { Pulmonary artery } \\
\text { pressure (mm Hg) }\end{array}$ & $0.29(0.08)$ & $0.40(0.037)$ & $0.47(0.008)$ & $0.39(0.02)$ & $0.53(0.002)$ & $0.36(<0.0001)$ \\
\hline $\begin{array}{l}\text { Valve area/EROA index } \\
\left(\mathrm{cm}^{2} / \mathrm{m}^{2}\right)\end{array}$ & - & $-0.28(0.10)$ & $-0.23(0.21)$ & $0.22(0.18)$ & $-0.05(0.79)$ & - \\
\hline $\begin{array}{l}\text { Exercise capacity } \\
\text { (METS) }\end{array}$ & $0.23(0.16)$ & $-0.44(0.009)$ & $-0.56(0.001)$ & $-0.11(0.52)$ & $-0.53(0.001)$ & $-0.38(<0.0001)$ \\
\hline
\end{tabular}


$\mathrm{p}<0.0001)$. Patients with plasma BNP concentrations $>42 \mathrm{pg} / \mathrm{mL}$ had a greater LAAI compared to those with a normal BNP in MS $(21 \pm 6$ vs $15 \pm 2 ; \mathrm{p}<0.001)$ and in AS $(13 \pm 3$ vs $11 \pm 2 ; \mathrm{p}<0.01)$. Despite no association with markers of LV size, there was a consistent correlation with LAAI across all valve lesions: MR $(\mathrm{r}=0.42, \mathrm{p}=0.02)$, MS $(\mathrm{r}=0.86, \mathrm{p}<0.0001), \quad \mathrm{AR} \quad(\mathrm{r}=0.53, \mathrm{p}=0.001), \quad$ AS $(\mathrm{r}=0.52, \mathrm{p}=0.002)$, all valves $(\mathrm{r}=0.57, \mathrm{p}<0.0001)$. An increase in plasma BNP concentration was also associated with a higher pulmonary artery pressure in all valve lesions.

\section{Exercise capacity and functional status}

Across all valve lesions, $55(40 \%)$ patients were symptomatic and were more likely to have a raised plasma BNP concentration than those who remained asymptomatic (49 (32-79) vs $31 \quad(22-44) \mathrm{pg} / \mathrm{mL} ; \mathrm{p}<0.001)$. Patients who were symptomatic had higher pulmonary artery pressures (38 \pm 12 vs $29 \pm 8 \mathrm{~mm} \mathrm{Hg} ; \mathrm{p}<0.001)$, larger LAAI ( $16 \pm 6$ vs $\left.14 \pm 5 \mathrm{~cm}^{2} / \mathrm{m}^{2} ; \mathrm{p}=0.02\right)$, and lower exercise capacity $(7.1 \pm 2.4$ vs $10.4 \pm 3.4$ metabolic equivalents (METS); $\mathrm{p}<0.001)$ than asymptomatic subjects. However, this finding was not consistent across all valve subgroups and, in particular, although asymptomatic patients with MS and AR had slightly higher exercise capacity than those with mild symptoms, this was not statistically significant (table 4). Patients with an elevated BNP performed less well on the treadmill than those with a normal BNP (10.1 \pm 3.1 vs $7.2 \pm 3.1$ METS, $\mathrm{p}<0.0001)$. This finding was consistent and statistically significant for all valve subgroups except for those with AR (table 2).

However, the patients with poor exercise capacity identified by NYHA class, and those by an elevated plasma BNP concentration, were not identical. Of the 81 patients in NYHA class 1, 20 patients had an elevated BNP. These patients performed less well on the treadmill than asymptomatic patients in whom BNP remained within the normal range $(10.9 \pm 3.1$ vs $8.7 \pm 3.8$ METS, $\mathrm{p}=0.01$ ).

\section{DISCUSSSION}

This study evaluated associations between the plasma BNP concentrations and echocardiographic measures of LV and LA remodelling in patients with moderate to severe aortic and mitral valve disease. Patients with

Table 4 Characteristics of asymptomatic versus symptomatic patients (mean \pm SD unless otherwise stated)

\begin{tabular}{|c|c|c|c|c|c|c|}
\hline & & Controls & $\begin{array}{l}\text { Aortic } \\
\text { stenosis }\end{array}$ & $\begin{array}{l}\text { Mitral } \\
\text { stenosis }\end{array}$ & $\begin{array}{l}\text { Aortic } \\
\text { regurgitation }\end{array}$ & $\begin{array}{l}\text { Mitral } \\
\text { regurgitation }\end{array}$ \\
\hline \multirow[t]{3}{*}{ Number of subjects } & NYHA 1 & 39 & 17 & 16 & 32 & 16 \\
\hline & NYHA 2 & & 17 & 14 & 7 & 17 \\
\hline & Total & 39 & 34 & 30 & 39 & 33 \\
\hline \multirow[t]{3}{*}{ BNP (pg/mL) } & NYHA 1 & $26.8 \pm 13.0$ & $33.7 \pm 19.3$ & $60.2 \pm 45.9$ & $40.9 \pm 37.7$ & $33.6 \pm 19.4$ \\
\hline & NYHA 2 & - & $45.0 \pm 24.8$ & $86.1 \pm 50.4$ & $74.2 \pm 75.9$ & $52.3 \pm 29.7$ \\
\hline & $\mathrm{p}$ Value & & 0.19 & 0.08 & 0.10 & 0.04 \\
\hline \multirow[t]{3}{*}{ Severe valve disease, $\mathrm{n}(\%)$} & NYHA 1 & - & $2(12)$ & $1(6)$ & $19(58)$ & $13(81)$ \\
\hline & NYHA 2 & - & $6(35)$ & $4(29)$ & $5(71)$ & $8(47)$ \\
\hline & $\mathrm{p}$ Value & & 0.22 & 0.16 & 0.69 & 0.04 \\
\hline \multirow[t]{3}{*}{ LV end-diastolic volume index $\left(\mathrm{mL} / \mathrm{m}^{2}\right)$} & NYHA 1 & $52.3 \pm 12.7$ & $52.8 \pm 14.4$ & $46.9 \pm 9.6$ & $90.3 \pm 28.6$ & $80.5 \pm 11.2$ \\
\hline & NYHA 2 & - & $46.6 \pm 18.1$ & $39.4 \pm 11.8$ & $95.7 \pm 29.5$ & $72.9 \pm 15.6$ \\
\hline & $\mathrm{p}$ Value & & 0.28 & 0.07 & 0.57 & 0.12 \\
\hline \multirow[t]{3}{*}{ LV end-systolic volume index $\left(\mathrm{mL} / \mathrm{m}^{2}\right)$} & NYHA 1 & $19.0 \pm 7.1$ & $18.7 \pm 7.6$ & $17.9 \pm 6.1$ & $33.3 \pm 13.7$ & $28.3 \pm 6.4$ \\
\hline & NYHA 2 & - & $22.8 \pm 15.4$ & $14.9 \pm 5.4$ & $36.7 \pm 16.8$ & $25.6 \pm 6.5$ \\
\hline & p Value & & 0.61 & 0.18 & 0.50 & 0.61 \\
\hline \multirow[t]{3}{*}{ LV ejection fraction (\%) } & NYHA 1 & $65 \pm 8$ & $63 \pm 8$ & $62 \pm 9$ & $64 \pm 6.2$ & $65 \pm 7$ \\
\hline & NYHA 2 & - & $61 \pm 6$ & $63 \pm 4$ & $63 \pm 7$ & $65 \pm 6$ \\
\hline & $\mathrm{p}$ Value & & 0.32 & 0.86 & 0.80 & 0.96 \\
\hline \multirow[t]{3}{*}{$\mathrm{LA}$ area index $\left(\mathrm{cm}^{2} / \mathrm{m}^{2}\right)$} & NYHA 1 & $10.6 \pm 1.9$ & $11.5 \pm 2.5$ & $18.2 \pm 6.0$ & $10.6 \pm 2.0$ & $17.4 \pm 3.9$ \\
\hline & NYHA 2 & - & $11.4 \pm 2.6$ & $20.5 \pm 5.9$ & $11.8 \pm 2.0$ & $17.6 \pm 5.0$ \\
\hline & $\mathrm{p}$ Value & & 0.90 & 0.16 & 0.14 & 0.73 \\
\hline \multirow[t]{3}{*}{ Pulmonary artery pressure $(\mathrm{mm} \mathrm{Hg})$} & NYHA 1 & $25 \pm 4$ & $30 \pm 7$ & $32 \pm 14$ & $28 \pm 6$ & $32 \pm 6$ \\
\hline & NYHA 2 & - & $35 \pm 6$ & $40 \pm 8$ & $31 \pm 9$ & $42 \pm 16$ \\
\hline & p Value & & 0.06 & 0.01 & 0.19 & 0.03 \\
\hline \multirow[t]{3}{*}{ Valve area index/EROA index $\left(\mathrm{cm}^{2} / \mathrm{m}^{2}\right)$} & NYHA 1 & - & $0.51 \pm 0.09$ & $0.71 \pm 0.15$ & $0.11 \pm 0.07$ & $0.38 \pm 0.16$ \\
\hline & NYHA 2 & - & $0.45 \pm 0.14$ & $0.62 \pm 0.16$ & $0.16 \pm 0.07$ & $0.29 \pm 0.15$ \\
\hline & $\mathrm{p}$ Value & & 0.15 & 0.13 & 0.06 & 0.10 \\
\hline \multirow[t]{3}{*}{ Exercise capacity (METS) } & NYHA 1 & $12.6 \pm 3.1$ & $10.9 \pm 2.8$ & $7.0 \pm 3.0$ & $11.3 \pm 3.6$ & $11.1 \pm 1.6$ \\
\hline & NYHA 2 & - & $7.4 \pm 2.5$ & $5.5 \pm 2.0$ & $9.4 \pm 1.9$ & $7.2 \pm 2.1$ \\
\hline & $\mathrm{p}$ Value & & 0.003 & 0.19 & 0.26 & $<0.0001$ \\
\hline
\end{tabular}

BNP, brain natriuretic peptide; EROA, effective regurgitant orifice area; LA, left atrium; LV, left ventricle; NYHA, New York Heart Association. 
impaired LVEF were excluded, so that the impact of factors other than LV systolic function could be evaluated. There were several clinically relevant observations. First, while there was a wide range of $\mathrm{LV}$ remodelling across different valve lesions, there was no correlation between the plasma BNP concentration and LV end systolic or end diastolic volumes for any valve lesion. This implies that in VHD patients with normal EF, compensatory $\mathrm{LV}$ remodelling is not associated with an increase in plasma BNP concentration. Second, the plasma BNP concentration correlated with LA size in all valve lesions. Third, plasma BNP concentrations were higher in subjects with mitral compared to aortic valve disease, and highest in patients with mitral stenosis, in which setting there is no increase in LV wall stretch or pressure load. BNP also increased with higher pulmonary artery pressures. In these patients, the elevation in pulmonary artery pressure was likely to be secondary to an increase in LA filling pressure. These observations are consistent with BNP release from the LA in response to an increase in volume and wall stretch. This hypothesis is supported by evidence for synthesis of BNP by atrial myocytes in response to chronic increases in wall stress, ${ }^{37}$ and costorage of BNP with ANP in atrial granules. ${ }^{38}$

In aortic valve disease, plasma BNP concentrations increased with relatively small increases in LA volume. It is possible that LA dilatation in aortic valve disease reflects subtle LV systolic or diastolic dysfunction. However, assessment of diastolic function, particularly in patients with mitral valve disease is not well validated, and comparison across valve lesions is not possible. In subjects with mitral valve disease, small increases in LA volume were often not associated with higher plasma BNP concentrations, but more marked LA enlargement usually was. Some patients with mitral stenosis were in $\mathrm{AF}$, which is known to be associated with higher plasma BNP concentrations. ${ }^{39} 40$ The number of patients with $\mathrm{AF}$ in this study was too small to allow a reliable analysis of the relationship between $\mathrm{AF}$ and $\mathrm{BNP}$.

Higher plasma BNP concentration was associated with pulmonary hypertension in all valve subgroups. In addition, higher BNP was associated with reduced exercise capacity in all valve lesions except for AR. Symptomatic patients with any valve lesion were more likely to have an elevated pulmonary artery pressure or a decreased exercise capacity. As this elevated pulmonary pressure and decreased exercise capacity are the objective correlates of symptomatic status, this is not surprising. However, there was considerable overlap in the objective parameters between symptomatic and asymptomatic patients consistent with the known difficulties in assessing cardiac symptoms particularly in the early stages of decompensation. ${ }^{41}$ Furthermore, although both symptomatic patients and those with elevated plasma BNP concentrations tended to have objective evidence of exercise limitation or echocardiographic features of more advanced valve disease, the patients identified were not identical. This suggests that plasma BNP concentrations may offer additional information in assessing the haemodynamic consequences of valve lesions in patients with AS, MS and MR. We did not find similar associations between BNP and exercise capacity in patients with AR. The reason for the lack of a statistically significant association in the AR cohort is uncertain.

\section{Clinical implications}

In this study, most patients with severe valve disease had a plasma BNP concentration of $<100 \mathrm{pg} / \mathrm{mL}$ : a value below which cardiac failure is considered unlikely in patients presenting with dyspnoea. ${ }^{42}$ Patients with valve disease and a preserved LVEF may therefore have clinically important symptoms and pulmonary hypertension with a relatively modest increase in BNP. Conversely, in patients with other reasons for an elevated BNP, measuring plasma BNP concentration to assess valve status may not be helpful. In this study, patients with other known causes of an elevated BNP were excluded.

Previous studies which evaluate outcomes in patients with mitral regurgitation ${ }^{13}$ and $\mathrm{AS}^{14}$ suggest that an elevated BNP may identify patients who have a higher risk of adverse events. There is known difficulty in evaluating symptoms in some patients, ${ }^{41}$ and a significant number of asymptomatic patients in this study had an elevated plasma BNP concentration. These patients had a lower exercise capacity than those in whom the BNP remained within the normal range. Consequently, elevated plasma BNP concentrations in apparently asymptomatic patients should lead to a more careful assessment regardless of the type of valve lesion.

\section{Study limitations}

The proportion of asymptomatic and symptomatic patients in each valve group differed, but except in the AR subgroup, at least half the patients in each valve type had mild or equivocal symptoms. The small number of symptomatic patients in the AR subgroup may partly explain the lack of associations between BNP and exercise capacity in this group. In the AS subgroup, only asymptomatic patients and those patients with equivocal symptoms underwent exercise testing, and in the other subgroups, patients had, at most, mild symptoms, as those with contraindications to exercise testing were excluded from the study. In particular, patients with severe AS with symptoms of angina were excluded from the study. This selection of predominantly asymptomatic and mildly symptomatic patients may be responsible for the relatively modest rise in plasma BNP concentration in this cohort. However, in clinical practice, patients with significant valve disease who have clear symptoms, usually have a class 1 indication for surgery or valve intervention according to current guidelines. ${ }^{21}{ }^{43}$ Consequently, the measurement of BNP in these patients is unlikely to be helpful. A further limitation is that although the overall number of patients within this study is modest, the number of patients within each valve group is relatively small. This limits the power of 
further subgroup analysis or comparison against other markers of valve severity within each subgroup. Furthermore, although valve severity was graded according to the American Society of Echocardiography/ European Society of Echocardiography guidelines,${ }^{23}$ the grading of severity, particularly between regurgitant and stenotic lesions differs considerably, and hence, is not directly comparable.

\section{CONCLUSION}

In patients with isolated heart valve disease and a preserved EF, significant cardiac remodelling can occur while BNP remains within the normal range. However, BNP increases with greater left atrial size and pulmonary artery pressure in all valve lesions. Consequently, plasma BNP concentrations cannot reliably identify patients with significant valve disease, and therefore, should be interpreted with caution in these patients. However, elevated BNP levels were associated with poor exercise capacity, and therefore, a raised BNP in an apparently asymptomatic patient with stable valve disease should prompt further assessment.

Acknowledgements The authors are very grateful to Dr Gillian Whalley for her assistance with left ventricular analysis, to Jenny White for coordinating the study, and to Margaret Oldfield for her expert echocardiography.

Contributors All authors have made a significant contribution to this original research article and reviewed the manuscript.

Funding Supported by grants from the New Zealand Heart Foundation, Middlemore Hospital Cardiac Trust (VS) and Green Lane Research and Education Fund (RAS); supported by grants from the New Zealand Heart Foundation and Green Lane Cardiovascular fund. DEN is supported by the British Heart Foundation (CH/09/002) and is the recipient of a Wellcome Trust Senior Investigator Award (WT103782AIA).

Competing interests None declared.

Ethics approval Northern X Ethics Committee, Auckland.

Provenance and peer review Not commissioned; externally peer reviewed.

Data sharing statement No additional data are available.

Open Access This is an Open Access article distributed in accordance with the Creative Commons Attribution Non Commercial (CC BY-NC 4.0) license, which permits others to distribute, remix, adapt, build upon this work noncommercially, and license their derivative works on different terms, provided the original work is properly cited and the use is non-commercial. See: http:// creativecommons.org/licenses/by-nc/4.0/

\section{REFERENCES}

1. Metra M, Nodari S, Parrinello G, et al. The role of plasma biomarkers in acute heart failure. Serial changes and independent prognostic value of NT-proBNP and cardiac troponin-T. Eur J Heart Fail 2007:9:776-86.

2. McDonagh TA, Cunningham AD, Morrison CE, et al. Left ventricular dysfunction, natriuretic peptides, and mortality in an urban population. Heart 2001;86:21-6.

3. Rodeheffer RJ. Measuring plasma B-type natriuretic peptide in heart failure: good to go in 2004? J Am Coll Cardiol 2004;44:740-9.

4. Richards AM, Nicholls MG, Yandle TG, et al. Plasma N-terminal pro-brain natriuretic peptide and adrenomedullin: new neurohormonal predictors of left ventricular function and prognosis after myocardial infarction. Circulation 1998;97:1921-9.
5. Sharma V, Stewart RA, Zeng I, et al. Comparison of atrial and brain natriuretic peptide for the assessment of mitral stenosis. Heart Lung Circ 2011;20:517-24.

6. Gerber IL, Stewart RA, Legget ME, et al. Increased plasma natriuretic peptide levels reflect symptom onset in aortic stenosis. Circulation 2003;107:1884-90.

7. Weber M, Arnold R, Rau M, et al. Relation of N-terminal pro-B-type natriuretic peptide to severity of valvular aortic stenosis. $A m \mathrm{~J}$ Cardiol 2004;94:740-5.

8. Gerber IL, Stewart RA, French JK, et al. Associations between plasma natriuretic peptide levels, symptoms, and left ventricular function in patients with chronic aortic regurgitation. $\mathrm{Am} \mathrm{J}$ Cardiol 2003:92:755-8.

9. Gabriel RS, Kerr AJ, Sharma V, et al. B-type natriuretic peptide and left ventricular dysfunction on exercise echocardiography in patients with chronic aortic regurgitation. Heart 2008;94:897-902.

10. Sutton TM, Stewart RA, Gerber IL, et al. Plasma natriuretic peptide levels increase with symptoms and severity of mitral regurgitation. $J$ Am Coll Cardiol 2003;41:2280-7.

11. Detaint D, Messika-Zeitoun D, Avierinos JF, et al. B-type natriuretic peptide in organic mitral regurgitation: determinants and impact on outcome. Circulation 2005;111:2391-7.

12. Arat-Ozkan A, Kaya A, Yigit Z, et al. Serum N-terminal pro-BNP levels correlate with symptoms and echocardiographic findings in patients with mitral stenosis. Echocardiography 2005;22:473-8.

13. Pizarro R, Bazzino OO, Oberti PF, et al. Prospective validation of the prognostic usefulness of brain natriuretic peptide in asymptomatic patients with chronic severe mitral regurgitation. J Am Coll Cardiol 2009:54:1099-106.

14. Lancellotti P, Moonen M, Magne J, et al. Prognostic effect of long-axis left ventricular dysfunction and B-type natriuretic peptide levels in asymptomatic aortic stenosis. Am J Cardiol 2010;105:383-8.

15. Detaint D, Messika-Zeitoun D, Chen $\mathrm{HH}$, et al. Association of B-type natriuretic peptide activation to left ventricular end-systolic remodeling in organic and functional mitral regurgitation. $\mathrm{Am} \mathrm{J}$ Cardiol 2006;97:1029-34

16. Nessmith MG, Fukuta $\mathrm{H}$, Brucks $\mathrm{S}$, et al. Usefulness of an Elevated B-Type Natriuretic Peptide in Predicting Survival in Patients With Aortic Stenosis Treated Without Surgery. Am J Cardiol 2005;96:1445-8.

17. Kerr AJ, Raffel OC, Whalley GA, et al. Elevated B-type natriuretic peptide despite normal left ventricular function on rest and exercise stress echocardiography in mitral regurgitation. Eur Heart $J$ 2008:29:363-70.

18. Van Pelt NC, Kerr AJ, Legget ME, et al. Increased B-type natriuretic peptide is associated with an abnormal blood pressure response to exercise in asymptomatic aortic stenosis. Int $\mathrm{J}$ Cardiol 2008;127:313-20.

19. Baumgartner $\mathrm{H}$, Hung J, Bermejo J, et al. Echocardiographic assessment of valve stenosis: EAE/ASE recommendations for clinical practice. Eur J Echocardiogr 2009;10:1-25.

20. Zoghbi WA, Enriquez-Sarano M, Foster E, et al. Recommendations for evaluation of the severity of native valvular regurgitation with two-dimensional and Doppler echocardiography. J Am Soc Echocardiogr 2003;16:777-802.

21. Bonow RO, Carabello BA, Kanu C, et al. ACC/AHA 2006 guidelines for the management of patients with valvular heart disease: a report of the American College of Cardiology/American Heart Association Task Force on Practice Guidelines. Circulation 2006;114:e84-231.

22. Coats AJ, Shewan LG. Statement on authorship and publishing ethics in the International Journal of Cardiology. Int J Cardiol 2011;153:239-40.

23. Baumgartner $\mathrm{H}$, Hung J, Bermejo J, et al. Echocardiographic assessment of valve stenosis: EAE/ASE recommendations for clinical practice. J Am Soc Echocardiogr 2009;22:1-23; quiz 101-2.

24. Leung DY, Griffin BP, Stewart WJ, et al. Left ventricular function after valve repair for chronic mitral regurgitation: predictive value of preoperative assessment of contractile reserve by exercise echocardiography. J Am Coll Cardiol 1996;28:1198-205.

25. Lester SJ, Ryan EW, Schiller NB, et al. Best method in clinical practice and in research studies to determine left atrial size. $A m \mathrm{~J}$ Cardiol 1999;84:829-32.

26. Enriquez-Sarano M, Bailey KR, Seward JB, et al. Quantitative Doppler assessment of valvular regurgitation. Circulation 1993;87:841-8.

27. Enriquez-Sarano M, Miller FA Jr, Hayes SN, et al. Effective mitral regurgitant orifice area: clinical use and pitfalls of the proximal isovelocity surface area method. J Am Coll Cardiol 1995;25:703-9.

28. Nakatani S, Masuyama T, Kodama K, et al. Value and limitations of Doppler echocardiography in the quantification of stenotic mitral 
valve area: comparison of the pressure half-time and the continuity equation methods. Circulation 1988;77:78-85.

29. Martin RP, Rakowski H, Kleiman JH, et al. Reliability and reproducibility of two dimensional echocardiograph measurement of the stenotic mitral valve orifice area. Am J Cardiol 1979;43:560-8.

30. Hatle L, Brubakk A, Tromsdal A, et al. Noninvasive assessment of pressure drop in mitral stenosis by Doppler ultrasound. $\mathrm{Br}$ Heart $J$ 1978;40:131-40.

31. Quinones MA, Otto CM, Stoddard M, et al. Recommendations for quantification of Doppler echocardiography: a report from the Doppler Quantification Task Force of the Nomenclature and Standards Committee of the American Society of Echocardiography. J Am Soc Echocardiogr 2002;15:167-84.

32. Himelman RB, Stulbarg M, Kircher B, et al. Noninvasive evaluation of pulmonary artery pressure during exercise by saline-enhanced Doppler echocardiography in chronic pulmonary disease. Circulation 1989;79:863-71.

33. Otto CM. Textbook of Clinical Echocardiography. Philadelphia, WB: Saunders, 2000.

34. Du Bois D, Du Bois EF. Clinical Calorimetry: Tenth Paper a Formula to Estimate the Approximate Surface Area if Height and Weight Be Known. Arch Intern Med 1916;17:863.

35. Yandle TG, Richards AM, Gilbert A, et al. Assay of brain natriuretic peptide (BNP) in human plasma: evidence for high molecular weight BNP as a major plasma component in heart failure. $J$ Clin Endocrinol Metab 1993;76:832-8.

36. Van Pelt NC, Stewart RAH, Legget ME, et al. Longitudinal left ventricular contractile dysfunction after exercise in aortic stenosis. Heart 2007;93:732-8.
37. Langenickel T, Pagel I, Hohnel K, et al. Differential regulation of cardiac ANP and BNP mRNA in different stages of experimental heart failure. Am J Physiol Heart Circ Physiol 2000;278:H1500-6.

38. Goetze JP, Friis-Hansen L, Rehfeld JF, et al. Atrial secretion of B-type natriuretic peptide. Eur Heart $J$ 2006;27:1648-50.

39. Dussaule JC, Vahanian A, Michel PL, et al. Plasma atrial natriuretic factor and cyclic GMP in mitral stenosis treated by balloon valvulotomy. Effect of atrial fibrillation. Circulation 1988;78:276-85.

40. Engelmann MDM, Niemann L, Kanstrup I-L, et al. Natriuretic peptide response to dynamic exercise in patients with atrial fibrillation. Int $J$ Cardiol 2005;105:31-9.

41. Messika-Zeitoun D, Johnson BD, Nkomo V, et al. Cardiopulmonary exercise testing determination of functional capacity in mitral regurgitation: physiologic and outcome implications. J Am Coll Cardiol 2006;47:2521-7.

42. Dickstein K, Cohen-Solal A, Filippatos G, et al., ESC Committee for Practice Guidelines (CPG). ESC guidelines for the diagnosis and treatment of acute and chronic heart failure 2008: the Task Force for the diagnosis and treatment of acute and chronic heart failure 2008 of the European Society of Cardiology. Developed in collaboration with the Heart Failure Association of the ESC (HFA) and endorsed by the European Society of Intensive Care Medicine (ESICM). Eur J Heart Fail 2008; 10:933-89.

43. Vahanian A, Baumgartner $\mathrm{H}, \mathrm{Bax} \mathrm{J}$, et al. Guidelines on the management of valvular heart disease: The Task Force on the Management of Valvular Heart Disease of the European Society of Cardiology. Eur Heart J 2007;28:230-68. 